Conclusion Taken together, these data show that alleles at class I loci are associated with the disease, although it is difficult to assess their specificity to PsA genetic susceptibility. No particular role of the HLA class II region could be demonstrated.

\section{SAT0024 ENTHESITIS AND DACTYLITIS AS THE BEGINNNIG STAGE OF PSORIATIC ARTHRITIS}

C Montilla, CM González, FJ López-Longo, M Moreno-Zazo, R Castillo, N Caro, L Cebrián, C González-Montagut, S Gómez, I Monteagudo, L Carreño. Rheumatology, Hospital General Universitario Gregorio Marañón, Madrid, Spain

\subsection{6/annrheumdis-2001.376}

Background Enthesitis and dactylitis are characteristic features of psoriatic arthritis and the others spondyloarthropathies. Both may appear from the onset of the disease.

Objectives To analize the clinical characteristic of a population of patients with psoriatic arthritis who presented enthesitis and dactylitis as their only clinical symptoms at the onset of the disease.

Methods We have studied a group of 204 patients diagnosed with psoriatic arthritis following the Moll and Wright criteria, selecting only those who expressed either Enthesitis or dactylitis as initial symptoms of the disease.

Results Twenty-eight patients (14\%) proved to have Enthesitis or dactylitis 21 enthesitis, 7 dactylitis and 1 Enthesitis and dactylitis as disease onset, and seventeen were men (60.7\%). The mean age of psoriatic arthritis onset was 38 years (range: 17-58). The mean time from Enthesitis and dactylitis onset and the Moll and Wright criteria was 6 months (range: 1-180). Enthesitis was most frequent in achilles tendon (81\%) and the plantar fascia (72.7\%). Twenty patients (71.4\%) presented arthritis involving peripheral joints, $4(14.3 \%)$ presented and axial skeleton distribution, and the remaining (14.3\%) showed a mixed peripheral and axial distribution.

Conclusion Enthesitis and dactylitis are initial clinical features of psoriatic arthritis, even though the majority of the patients with these symptoms at the beginning of the disease evolve towards a peripheral arthritis pattern.

\section{SAT0025 EVOLUTION OF PSORIATIC ARTHRITIS WITH DISTAL INTERPHALANGEAL JOINTS INVOLVEMENT AT ONSET}

CA Montilla, CM González, FJ López-Longo, R Castillo, N Caro, C González-Montagut, L Cebrián, S Gómez, E Carmona, I Monteagudo, L Carreño. Rheumatology, Hospital General Universitario Gregorio Marañón, Madrid, Spain

10.1136/annrheumdis-2001.377

Background Distal interphalangeal joints (DIP) arthritis described by Moll and Wright as a psoriatic arthritis (Psa) pattern is now considered an evolutionary step towards other classification patterns.

Objectives To study the clinical and radiological evolution of a group of patients with arthritis limited to the DIP as onset of Psa.

Methods We have studied the clinical and radiological evolution of 204 patients diagnosed with Psa (Moll and Wright criteria) during the first year of the disease selecting those patients whose initial clinical features were exclusively arthritis of the DIP. The radiological findings after one-year follow-up were classified into stages according to Steinbrocker's criteria.
Results Arthritis of DIP as onset of the disease was found in 19/ $204(9.3 \%)$ of the patients. The mean age at onset was 49 years (range: 34-64). Psoriasis before arthritis onset was present in 8/ $19(42.1 \%)$ and in 9/19 (47.3\%) the onset of arthritis and psoriasis was simultaneous. Onychodystrophy (pitting) was present in $7 / 19$ (36.8\%). After one-years 9/19 (47.3\%) patients continued with arthritis as the only manifestations of Psa, 8/19 (42.1\%) patients evolved toward other arthritis patterns (5 asymmetrical oligoarthritis and 3 symmetrical polyarthritis). The joints more commonly involved along with the DIP were metacarpophalangeal $(75 \%)$ and proximal interphalangeal (62.5\%). 2/19 patients $(10.4 \%)$ did not show DIP arthritis after one year. At the end of the one-year study $9 / 19(47,3 \%)$ of the patients were classified into III stage radiological criteria, 2/19 (10.4\%) were at the stage $\mathrm{I}$ and in the remaining patients radiological was considered as normal.

Conclusion Almost half of the patients with DIP arthritis as Psa onset evolve within one year towards others arthritis patterns. Half of the patients revealed an aggressive radiological progression during the first year of the disease.

\section{SAT0026 IS RADIOGRAPHIC EXAMINATION SUFFICIENT FOR DETECTION OF NON TRAUMATIC SPINAL FRACTURES IN ANKYLOSIG SPONDYLITIS: COMPARISON OF RADIOGRAPHIC AND MRI FINDINGS}

${ }^{1} \mathrm{H}$ Przepiera-Bedzak, ${ }^{2}$ L Cyrylowski, ${ }^{1} \mathrm{M}$ Brzosko. ${ }^{1}$ Rheumatology; ${ }^{2}$ Radiology, Pomeranian Academy of Medicine, Szczecin, Poland

10.1136/annrheumdis-2001.378

\section{Background}

Objectives To compare plain radiographic and MRI findings in evaluation of non traumatic spinal fractures in patients with ankylosing spondylitis (AS), and to find correlation between spinal fractures and disease activity, age of patients, and disease duration.

Methods 21 males and 2 females with AS, mean aged 49.8, were studied. Lateral radiographs and MRI examinations of lumbar, thoracic and cervical spine were performed in all patients. To detect vertebral body fractures with radiographs, the anterior, posterior and central heights of each vertebral body were measured. The ratio of each height to that of the two adjacent vertebrae, the ratio of the anterior height to the posterior height and of the central height to the posterior height were calculated. The subjects were considered to have fracture if any of these ratios was less than 0,85 for lumbar spine and less than 0,8 for thoracic and cervical spine. We assessed also the following indexes of disease activity: BASFI, BASDAI, BASG-t, BASG-6, BSMI.

Results Total 25 fractures were found with the radiographic method: 7 fractures in lumbar spine, 15 in thoracic and 3 in cervical. The MRI examinations revealed total 51 fractures: 24 fractures in lumbar spine, 18 in thoracic and 9 in cervical. Out of 25 fractures detected by X-ray examination, MRI confirmed the site of fracture only in $10(40 \%)$. Age of patients and disease duration correlated positively with total number of fractures, number of fractures in lumbar spine and number of fractures in thoracic detected by MRI. There was no such correlation for fractures detected by X-ray. Assessing indexes of disease activity we found positive correlation between BASFI, BASDAI, BASMI and total number of fractures and number of fractures in lumbar spine detected by MRI. 